\title{
At least three solutions for the Hamiltonian system and reduction method
}

\author{
Tacksun Jung ${ }^{1}$ and Q-Heung Choi ${ }^{2 *}$
}

${ }^{\text {*Correspondence: }}$
qheung@inha.ac.kr
${ }^{2}$ Department of Mathematics
Education, Inha University, Incheon,
$402-751$, Korea
Full list of author information is
available at the end of the article

available at the end of the article

\begin{abstract}
We investigate the multiplicity of solutions for the Hamiltonian system with some asymptotically linear conditions. We get a theorem which shows the existence of at least three $2 \pi$-periodic solutions for the asymptotically linear Hamiltonian system. We obtain this result by the variational reduction method which reduces the infinite dimensional problem to the finite dimensional one. We also use the critical point theory and the variational method.
\end{abstract}

MSC: 35A15; 37K05

Keywords: Hamiltonian system; asymptotical linearity; variational reduction method; critical point theory; variational method; (P.S.) $)_{c}$ condition

\section{Introduction and statement of the main result}

Let $G(t, z(t))$ be a $C^{2}$ function defined on $R^{1} \times R^{2 n}$ which is $2 \pi$-periodic with respect to the first variable $t$. In this paper we investigate the number of $2 \pi$-periodic solutions of the following Hamiltonian system:

$$
\begin{aligned}
& \dot{p}(t)=-G_{q}(t, p(t), q(t)), \\
& \dot{q}(t)=G_{p}(t, p(t), q(t)),
\end{aligned}
$$

where $p, q \in R^{n}, z=(p, q)$. Let $J$ be the standard symplectic structure on $R^{2 n}$, i.e.,

$$
J=\left(\begin{array}{cc}
0 & -I_{n} \\
I_{n} & 0
\end{array}\right)
$$

where $I_{n}$ is the $n \times n$ identity matrix. Then (1.1) can be rewritten as

$$
-J \dot{z}=G_{z}(t, z(t))
$$

where $\dot{z}=\frac{d z}{d t}$ and $G_{z}$ is the gradient of $G$. We assume that $G \in C^{2}\left(R^{1} \times R^{2 n}, R^{1}\right)$ satisfies the following asymptotically linear conditions:

(G1) $G(t, z(t))=o\left(|z|^{2}\right)$ as $|z| \rightarrow 0, G(t, \theta)=0, G_{z}(t, \theta)=\theta$, where $\theta=(0, \ldots, 0)$.

(G2) There exist constants $\alpha, \beta$ (without loss of generality, we may assume $\alpha, \beta \notin Z$ ) such that

$$
\alpha I \leq d_{z}^{2} G(t, z) \leq \beta I \quad \forall(t, z) \in R^{1} \times R^{2 n}
$$


(G3) Let $j_{1}$ be an integer within $[\alpha, \beta]$ such that

$$
j_{1}-1<\alpha<d_{z}^{2} G(t, 0)=\lim _{|z| \rightarrow 0} \frac{G_{z}(t, z) \cdot z}{|z|^{2}}<j_{1}
$$

(G4) $\lim _{|z| \rightarrow \infty} \frac{G_{z}(t, z) \cdot z}{|z|^{2}}$ exists and there exists $j_{2}=j_{1}+1$ which satisfies

$$
j_{1}<d_{z}^{2} G(t, \infty)=\lim _{|z| \rightarrow \infty} \frac{G_{z}(t, z) \cdot z}{|z|^{2}}<\beta<j_{2}
$$

(G5) $G$ is $2 \pi$-periodic with respect to $t$.

We are looking for the weak solutions of (1.1) with conditions (G1)-(G5). The $2 \pi$-periodic weak solution $z=(p, q) \in E$ of (1.1) satisfies

$$
\int_{0}^{2 \pi}\left(\dot{z}-J\left(G_{z}(t, z(t))\right)\right) \cdot J w d t=0 \quad \text { for all } w \in E
$$

i.e.,

$$
\int_{0}^{2 \pi}\left[\left(\dot{p}+G_{q}(t, z(t))\right) \cdot \psi-\left(\dot{q}-G_{p}(t, z(t))\right) \cdot \phi\right] d t=0 \quad \text { for all } \zeta=(\phi, \psi) \in E,
$$

where $E$ is introduced in Section 2. By Lemma 2.1 in Section 2, the weak solutions of (1.1) coincide with the critical points of the functional

$$
f(z)=\frac{1}{2} \int_{0}^{2 \pi}(-J \dot{z}) \cdot z d t-\int_{0}^{2 \pi} G(t, z(t)) d t=\int_{0}^{2 \pi} p \dot{q} d t-\int_{0}^{2 \pi} G(t, z(t)) d t .
$$

Several authors [1-4] considered the multiplicity of solutions for the Hamiltonian system. Chang proved in [1] that if $G \in C^{2}\left(R^{1} \times R^{2 n}, R^{1}\right)$ satisfies conditions (G2), (G5) and the following additional conditions:

$(\mathrm{G} 3)^{\prime}$ Let $j_{0}, j_{0}+1, \ldots$, and $j_{1}$ be all integers within $[\alpha, \beta]$ (without loss of generality, we may assume $\alpha, \beta \notin Z$ ) such that $j_{0}-1<\alpha<j_{0}<j_{1}<\beta<j_{1}+1=j_{2}$. Suppose that there exist $\gamma>0$ and $\tau>0$ such that $j_{1}<\gamma<\beta$ and

$$
G(t, z) \geq \frac{1}{2} \gamma\|z\|_{L^{2}}^{2}-\tau \quad \forall(t, z) \in R^{1} \times R^{2 n}
$$

$(\mathrm{G} 4)^{\prime} G_{z}(t, \theta)=\theta$ and $j \in\left[j_{0}, j_{1}\right) \cap Z$ such that

$$
j I<d_{z}^{2} G(t, \theta)<(j+1) I \quad \forall t \in R^{1},
$$

then (1.1) has at least two nontrivial $2 \pi$-periodic weak solutions. Jung and Choi proved in [2] that if $G$ satisfies the following conditions:

$(\mathrm{G} 1)^{\prime \prime} \quad G: R^{2 n} \rightarrow R$ is $C^{1}$ with $G(\theta)=0$.

(G2)" There exists $h \in N$ such that

$$
h<\lim \inf _{|z| \rightarrow \infty} \frac{G^{\prime}(z) \cdot z}{|z|^{2}}<h+1 .
$$


$(\mathrm{G} 3)^{\prime \prime}$ There exists $m \in N$ such that

$$
h+2 m<\lim \inf _{|z| \rightarrow 0} \frac{G^{\prime}(z) \cdot z}{|z|^{2}}<h+2 m+1
$$

or

$$
h-2 m-1<\lim \sup _{|z| \rightarrow 0} \frac{G^{\prime}(z) \cdot z}{|z|^{2}}<h-2 m .
$$

$(\mathrm{G} 4)^{\prime \prime}$ There exists an integer $\Gamma$ such that $\Gamma \leq \frac{G^{\prime}(z) \cdot z}{|z|^{2}} \leq \Gamma+1$,

then (1.1) has at least $m$ weak solutions, which are geometrically distinct and nonconstant.

Our main result is the following:

Theorem 1.1 Assume that G satisfies conditions (G1)-(G5). Then system (1.1) has at least three $2 \pi$-periodic solutions.

Theorem 1.1 will be proved by the finite dimensional reduction method, the critical point theory and the variational method for the perturbed operator $A_{\epsilon}$. The finite dimensional reduction method combined with the critical point theory and the variational method reduces the critical point results of the functional $I(z)$ on the infinite dimensional space to those of the corresponding functional $\tilde{I}(v)$ on the finite dimensional subspace.

The outline of this paper is organized as follows. In Section 2, we introduce the Hilbert normed space $E$, show that the corresponding functional $I(z)$ of $(1.1)$ is in $C^{1}(E, R)$, Fréchet differentiable and prove the reduction lemma for the perturbed operator $A_{\epsilon}$. In Section 3, we show that the reduced functional $-\tilde{I}(v)$ satisfies $(P . S .)_{c}$ condition and $v=0$ is the strict local point of minimum of $\tilde{I}(v)$ and prove Theorem 1.1 by the shape of graph of the reduced functional.

\section{The perturbed operator $A_{\epsilon}$}

Let $L^{2}\left([0,2 \pi], R^{2 n}\right)$ denote the set of $2 n$-tuples of the square integrable $2 \pi$-periodic functions and choose $z \in L^{2}\left([0,2 \pi], R^{2 n}\right)$. Then it has a Fourier expansion $z(t)=\sum_{k=-\infty}^{k=+\infty} a_{k} e^{i k t}$, with $a_{k}=\frac{1}{2 \pi} \int_{0}^{2 \pi} z(t) e^{-i k t} d t \in C^{2 n}, a_{-k}=\bar{a}_{k}$ and $\sum_{k \in Z}\left|a_{k}\right|^{2}<\infty$. Let

$$
A: z(t) \mapsto-J \dot{z}(t)
$$

with the domain

$$
\begin{aligned}
D(A) & =\left\{z(t) \in H^{1}\left([0,2 \pi], R^{2 n}\right) \mid z(0)=z(2 \pi)\right\} \\
& =\left\{\left.z(t) \in L^{2}\left([0,2 \pi], R^{2 n}\right)\left|\sum_{k \in Z}(\epsilon+|k|)^{2}\right| a_{k}\right|^{2}<+\infty\right\},
\end{aligned}
$$

where $\epsilon$ is a positive small number. Then $A$ is a self-adjoint operator. Let $\left\{M_{\lambda}\right\}$ be the spectral resolution of $A$, and let

$$
P_{0}=\int_{\alpha}^{\beta} d M_{\lambda}, \quad P_{+}=\int_{\beta}^{+\infty} d M_{\lambda}, \quad P_{-}=\int_{-\infty}^{\alpha} d M_{\lambda}
$$


Let

$$
L_{0}=P_{0} L^{2}\left([0,2 \pi], R^{2 n}\right), \quad L_{+}=P_{+} L^{2}\left([0,2 \pi], R^{2 n}\right), \quad L_{-}=P_{-} L^{2}\left([0,2 \pi], R^{2 n}\right) .
$$

For each $u \in L^{2}\left([0,2 \pi], R^{2 n}\right)$, we have the composition

$$
u=u_{0}+u_{+}+u_{-}
$$

where $u_{0} \in L_{0}, u_{+} \in L_{+}, u_{-} \in L_{-}$. According to $A$, there exists a small number $\epsilon>0$ such that $-\epsilon \notin \sigma(A)$. Let us define the space $E$ as follows:

$$
E=D\left(|A|^{\frac{1}{2}}\right)=\left\{\left.z \in L^{2}\left([0,2 \pi], R^{2 n}\right)\left|\sum_{k \in Z}(\epsilon+|k|)\right| a_{k}\right|^{2}<\infty\right\}
$$

with the scalar product

$$
(z, w)_{E}=\epsilon(z, w)_{L^{2}}+\left(|A|^{\frac{1}{2}} z,|A|^{\frac{1}{2}} w\right)_{L^{2}}
$$

and the norm

$$
\|z\|=(z, z)_{E}^{\frac{1}{2}}=\left(\sum_{k \in Z}(\epsilon+|k|)\left|a_{k}\right|^{2}\right)^{\frac{1}{2}} .
$$

The space $E$ endowed with this norm is a real Hilbert space continuously embedded in $L^{2}\left([0,2 \pi], R^{2 n}\right)$. The scalar product in $L^{2}$ naturally extends as the duality pairing between $E$ and $E^{\prime}=W^{-\frac{1}{2}, 2}\left([0,2 \pi], R^{2 n}\right)$. We note that the operator $(\epsilon+|A|)^{-1}$ is a compact linear operator from $L^{2}\left([0,2 \pi], R^{2 n}\right)$ to $E$ such that

$$
\left((\epsilon+|A|)^{-1} w, z\right)_{E}=(w, z)_{L^{2}} .
$$

Let

$$
A_{\epsilon}=\epsilon I+A \text {. }
$$

Let

$$
E_{0}=\left|A_{\epsilon}\right|^{-\frac{1}{2}} L_{0}, \quad E_{+}=\left|A_{\epsilon}\right|^{-\frac{1}{2}} L_{+}, \quad E_{-}=\left|A_{\epsilon}\right|^{-\frac{1}{2}} L_{-} .
$$

Then $E=E_{0} \oplus E_{+} \oplus E_{-}$and for $z \in E$, $z$ has the decomposition $z=z_{0}+z_{+}+z_{-} \in E$, where

$$
z_{0}=\left|A_{\epsilon}\right|^{-\frac{1}{2}} u_{0}, \quad z_{+}=\left|A_{\epsilon}\right|^{-\frac{1}{2}} u_{+}, \quad z_{-}=\left|A_{\epsilon}\right|^{-\frac{1}{2}} u_{-} .
$$

Thus we have

$$
\left\|z_{0}\right\|_{E_{0}}=\left\|u_{0}\right\|_{L_{0}}, \quad\left\|z_{+}\right\|_{E_{+}}=\left\|u_{+}\right\|_{L_{+}}, \quad\left\|z_{-}\right\|_{E_{-}}=\left\|u_{-}\right\|_{L_{-}}
$$


and that $E_{0}, E_{+}, E_{-}$are isomorphic to $L_{0}, L_{+}, L_{-}$, respectively. Let us define the functional $f(u)$ on $L^{2}$ as follows:

$$
f(u)=\frac{1}{2}\left(\left\|u_{+}\right\|^{2}+\left\|M_{+} u_{0}\right\|^{2}-\left\|M_{-} u_{0}\right\|^{2}-\left\|u_{-}\right\|^{2}\right)-\psi_{\epsilon}(z)
$$

where $M_{+}=\int_{0}^{\infty} d M_{\lambda}, M_{-}=\int_{-\infty}^{0} d M_{\lambda}$ and $\psi_{\epsilon}(z)=\psi(z)+\frac{\epsilon}{2}\|z(t)\|_{L^{2}}^{2}, \psi(z)=\int_{0}^{2 \pi} G(t, z(t)) d t$. Let

$$
F(z)=G_{z}(t, z(t))
$$

By $G \in C^{2}$ and (G2), $\psi(z)=\int_{0}^{2 \pi} G(t, z(t)) \in C^{2}\left(E, R^{1}\right)$. Let

$$
F_{\epsilon}(z)=\epsilon I+F(z)=\epsilon I+G_{z}(t, z(t)) .
$$

The system (1.1) is equal to

$$
A_{\epsilon}(z)=F_{\epsilon}(z) .
$$

The Euler equation of the functional $f(u)$ is the system

$$
\begin{aligned}
& u_{+}=\left|A_{\epsilon}\right|^{-\frac{1}{2}} P_{+} F_{\epsilon}(z), \\
& u_{-}=-\left|A_{\epsilon}\right|^{-\frac{1}{2}} P_{-} F_{\epsilon}(z), \\
& M_{+} u_{0}=\left|A_{\epsilon}\right|^{-\frac{1}{2}} M_{+} P_{0} F_{\epsilon}(z), \quad M_{-} u_{0}=-\left|A_{\epsilon}\right|^{-\frac{1}{2}} M_{-} P_{0} F_{\epsilon}(z) .
\end{aligned}
$$

Thus $z=z_{0}+z_{+}+z_{-}$is a solution of (2.2) if and only if $u=u_{0}+u_{+}+u_{-}$is a critical point of $f$. System (2.3)-(2.5) is reduced to

$$
\begin{aligned}
& A_{\epsilon} z_{+}=P_{+} F_{\epsilon}\left(z_{0}+z_{+}+z_{-}\right) \quad \text { or } \quad z_{+}=\left(A_{\epsilon}\right)^{-1} P_{+} F_{\epsilon}\left(z_{0}+z_{+}+z_{-}\right), \\
& A_{\epsilon} z_{-}=P_{-} F_{\epsilon}\left(z_{0}+z_{+}+z_{-}\right) \quad \text { or } \quad z_{-}=\left(A_{\epsilon}\right)^{-1} P_{-} F_{\epsilon}\left(z_{0}+z_{+}+z_{-}\right), \\
& A_{\epsilon} M_{+} z_{0}=M_{+} P_{0} F_{\epsilon}\left(z_{0}+z_{+}+z_{-}\right), \quad A_{\epsilon} M_{-} z_{0}=M_{-} P_{0} F_{\epsilon}\left(z_{0}+z_{+}+z_{-}\right) .
\end{aligned}
$$

By (G2),

$$
\left\|F_{\epsilon}(u)-F_{\epsilon}(v)\right\|_{L^{2}} \leq(\epsilon+\beta)\|u-v\|_{L^{2}} \quad \forall u, v \in L^{2} .
$$

By (G2), there exists a $\gamma>\beta+\epsilon$ such that

$$
\left\|\left.A_{\epsilon}^{-1}\right|_{L_{+} \oplus L_{-}}\right\| \leq \frac{1}{\gamma}
$$

We note that

$$
f(u)=f\left(u\left(z_{0}+z_{-}+z_{+}\right)\right) .
$$


Let us set

$$
I\left(z_{0}+z_{+}+z_{-}\right)=f\left(u\left(z_{0}+z_{-}+z_{+}\right)\right) \text {. }
$$

Now we will prove a reduction lemma which reduces the problem on the infinite dimensional space $E$ to that of the finite dimensional subspace.

Let $z_{0} \in E_{0}$ be fixed and consider the function $h: E_{-} \times E_{+} \rightarrow R$ defined by

$$
h\left(z_{-}, z_{+}\right)=I\left(z_{0}+z_{-}+z_{+}\right) .
$$

The function $h$ has continuous partial Fréchet derivatives $D_{1} h$ and $D_{2} h$ with respect to its first and second variables given by

$$
D_{i} h\left(z_{-}, z_{+}\right)\left(y_{i}\right)=D I\left(z_{0}+z_{-}+z_{+}\right)\left(y_{i}\right)
$$

for $y_{1} \in E_{-}$and $y_{2} \in E_{+}, i=1,2$. Let $v=z_{0}$.

Lemma 2.1 Assume that $G$ satisfies the conditions (G1)-(G5).

(i) For given $v \in E_{0}$, there exists a unique $z_{-}+z_{+} \in C^{1}\left(E_{0}, E_{-} \oplus E_{+}\right)$satisfying the equation

$$
A_{\epsilon}\left(z_{-}+z_{+}\right)=\left(P_{-}+P_{+}\right) F_{\epsilon}\left(v+z_{-}+z_{+}\right)
$$

(ii) There exists $m_{1}<0$ such that if $z_{-}$and $y_{-}$are in $E_{-}$and $z_{+} \in E_{+}$, then

$$
\left(D_{1} h\left(z_{-}, z_{+}\right)-D_{1} h\left(y_{-}, z_{+}\right)\right)\left(z_{-}-y_{-}\right) \leq m_{1}\left\|z_{-}-y_{-}\right\|^{2}
$$

(iii) There exists $m_{2}>0$ such that if $z_{+}$and $y_{+}$are in $E_{+}$and $z_{-} \in E_{-}$, then

$$
\left(D_{2} h\left(z_{-}, z_{+}\right)-D_{2} h\left(z_{-}, y_{+}\right)\right)\left(z_{+}-y_{+}\right) \geq m_{2}\left\|z_{+}-y_{+}\right\|^{2}
$$

(iv) For given $v \in E_{0}$, if we put the unique solution $z_{-}(v)+z_{+}(v)$ of $(2.11)$ as $z_{-}(v)+z_{+}(v)=\theta(v)$, then $\theta(v)$ is continuous on $E_{0}$ and satisfies a uniform Lipschitz condition in $E_{0}$ with respect to $L^{2}$ norm (also norm $\|\cdot\|_{E}$ ) and $\left|A_{\epsilon}\right|^{\frac{1}{2}} z_{-}(v) \in C^{1}\left(E_{0}, E_{-} \oplus E_{+}\right),\left|A_{\epsilon}\right|^{\frac{1}{2}} z_{+}(v) \in C^{1}\left(E_{0}, E_{-} \oplus E_{+}\right)$. Moreover,

$$
D I(v+\theta(v))(w)=0 \quad \text { for all } w \in E_{-} \oplus E_{+} .
$$

(v) If $\tilde{I}: E_{0} \rightarrow R$ is defined by

$$
\tilde{I}(v)=I(v+\theta(v))=I\left(v+z_{-}(v)+z_{+}(v)\right),
$$

then $\tilde{I}$ has a continuous Fréchet derivative DĨ with respect to $v$, and

$$
D \tilde{I}(v)(y)=D I(v+\theta(v))(y) \quad \text { for all } v, y \in E_{0} .
$$


(vi) $v \in E_{0}$ is a critical point of I if and only if $v+\theta(v)=v+z_{-}(v)+z_{+}(v)$ is a critical point of $I$.

Proof (i) Let $\delta=\frac{\alpha+\beta}{2}+\epsilon$. If $F_{\epsilon}^{\delta}(\psi)=F_{\epsilon}(\psi)-\delta$, then equation (2.11) is equivalent to the equation

$$
z_{-}+z_{+}=\left(A_{\epsilon}-\delta\right)^{-1}\left(P_{-}+P_{+}\right) F_{\epsilon}^{\delta}\left(v+z_{-}+z_{+}\right)
$$

The operator $\left(A_{\epsilon}-\delta\right)^{-1}\left(P_{-}+P_{+}\right)$is a self-adjoint, compact and linear map from $\left(P_{-}+P_{+}\right) L_{2}$ into itself and its norm is $\left(\min \left\{\left|j_{2}-\delta\right|,\left|j_{1}-1-\delta\right|\right\}\right)^{-1}$. We note that

$$
\begin{aligned}
\left\|F_{\epsilon}^{\delta}\left(\psi_{2}\right)-F_{\epsilon}^{\delta}\left(\psi_{1}\right)\right\|_{L^{2}} & \leq(\max \{|\alpha-\delta|,|\beta-\delta|\}+\epsilon)\left\|\psi_{2}-\psi_{1}\right\|_{L^{2}} \\
& =\left(\frac{\beta-\alpha}{2}+\epsilon\right)\left\|\psi_{2}-\psi_{1}\right\|_{L^{2}} .
\end{aligned}
$$

We claim that the right-hand side of (2.14) is a Lipschitz mapping of $\left(P_{-}+P_{+}\right) L_{2}$ into itself with a Lipschitz constant $r<1$. In fact, let $v$ be a fixed element in $E_{0}$ and $w=v+z_{-}+z_{+}$, $y=v+w_{-}+w_{+}$be any elements in $E$. Then we have

$$
\begin{aligned}
& \left\|\left(A_{\epsilon}-\delta\right)^{-1}\left(P_{-}+P_{+}\right) F_{\epsilon}^{\delta}\left(v+z_{-}+z_{+}\right)-\left(A_{\epsilon}-\delta\right)^{-1}\left(P_{-}+P_{+}\right) F_{\epsilon}^{\delta}\left(v+w_{-}+w_{+}\right)\right\|_{E} \\
& \quad=\left\|\left|A_{\epsilon}-\delta\right|^{-\frac{1}{2}}\left(P_{-}+P_{+}\right)\left(F_{\epsilon}^{\delta}(w)-F_{\epsilon}^{\delta}(y)\right)\right\|_{L^{2}} \\
& \quad \leq\left\|\left|A_{\epsilon}-\delta\right|^{-\frac{1}{2}}\left(P_{-}+P_{+}\right)\right\|\left\|\left(F_{\epsilon}^{\delta}(w)-F_{\epsilon}^{\delta}(y)\right)\right\|_{L^{2}} \\
& \quad \leq(\max \{|\alpha-\delta|,|\beta-\delta|\}+\epsilon)\left\|\left|A_{\epsilon}-\delta\right|^{-\frac{1}{2}}\left(P_{-}+P_{+}\right)\right\|\left\|\left(z_{-}+z_{+}\right)-\left(w_{-}+w_{+}\right)\right\|_{L^{2}} .
\end{aligned}
$$

Since the operator norm of $\left|A_{\epsilon}-\delta\right|^{-\frac{1}{2}}\left(P_{-}+P_{+}\right)$is less than or equal to $\frac{1}{\sqrt{\min \left\{\left|j_{2}-\delta\right|,\left|j_{1}-1-\delta\right|\right\}}+\epsilon}$ and

$$
\begin{aligned}
\left\|z_{-}\right\|_{L^{2}} & =\left\|\left|A_{\epsilon}\right|^{-\frac{1}{2}} u_{-}\right\|_{L^{2}} \leq \frac{1}{\sqrt{\min \left\{\left|j_{2}-\delta\right|,\left|j_{1}-1-\delta\right|\right\}}+\epsilon}\left\|u_{-}\right\|_{L^{2}} \\
& =\frac{1}{\sqrt{\min \left\{\left|j_{2}-\delta\right|,\left|j_{1}-1-\delta\right|\right\}}+\epsilon}\left\|z_{-}\right\|_{E}, \\
\left\|z_{+}\right\|_{L^{2}} & =\left\|\left|A_{\epsilon}\right|^{-\frac{1}{2}} u_{+}\right\|_{L^{2}} \leq \frac{1}{\sqrt{\min \left\{\left|j_{2}-\delta\right|,\left|j_{1}-1-\delta\right|\right\}}+\epsilon}\left\|u_{+}\right\|_{L^{2}} \\
& =\frac{1}{\sqrt{\min \left\{\left|j_{2}-\delta\right|,\left|j_{1}-1-\delta\right|\right\}}+\epsilon}\left\|z_{+}\right\|_{E},
\end{aligned}
$$

we have

$$
\begin{aligned}
& \left\|\left(A_{\epsilon}-\delta\right)^{-1}\left(P_{-}+P_{+}\right) F_{\epsilon}^{\delta}(w)-\left(A_{\epsilon}-\delta\right)^{-1}\left(P_{-}+P_{+}\right) F_{\epsilon}^{\delta}(y)\right\|_{E} \\
& \leq \frac{\max \{|\alpha-\delta|,|\beta-\delta|\}+\epsilon}{\min \left\{\left|j_{2}-\delta\right|,\left|j_{1}-1-\delta\right|\right\}+\epsilon}\left\|\left(z_{-}+z_{+}\right)-\left(w_{-}+w_{+}\right)\right\|_{E_{-} \oplus E_{+}} \\
& \quad=r\left\|\left(z_{-}+z_{+}\right)-\left(w_{-}+w_{+}\right)\right\|_{E_{-} \oplus E_{+}}, \quad \text { where } r<1,
\end{aligned}
$$


since $\min \left\{\left|j_{2}-\delta\right|,\left|j_{1}-1-\delta\right|\right\}+\epsilon>\max \{|\alpha-\delta|,|\beta-\delta|\}+\epsilon$. Therefore, by the implicit function theorem, for given $v \in E_{0}$, there exists a unique solution $z_{-}(v)+z_{+}(v) \in E_{-} \oplus E_{+}$ which satisfies (2.15).

(ii) For all $z_{-} \in E_{-}$,

$$
\left\|z_{-}\right\|_{E}^{2} \leq\left(j_{1}-1\right)\left\|w_{1}\right\|_{L^{2}}^{2}
$$

For all $z_{+} \in E_{+}$,

$$
\left\|z_{+}\right\|_{E}^{2} \geq\left(j_{1}+1\right)\left\|w_{1}\right\|_{L^{2}(\Omega)}^{2}
$$

If $v \in E_{0}, z_{-}$and $y_{-}$are in $E_{-}, z_{+} \in E_{+}$and $z=v+z_{-}+z_{+}$, then

$$
\begin{aligned}
& \left(D_{1} h\left(z_{-}, z_{+}\right)-D_{1} h\left(y_{-}, z_{+}\right)\right)\left(z_{-}-y_{-}\right) \\
& \quad=\int_{0}^{2 \pi}\left[A_{\epsilon}\left(z_{-}-y_{-}\right)\left(z_{-}-y_{-}\right)-\left(G_{z}^{\epsilon}\left(v+z_{-}+z_{+}\right)-G_{z}^{\epsilon}\left(v+y_{-}+z_{+}\right)\right)\left(z_{-}-y_{-}\right)\right] d t .
\end{aligned}
$$

Since $\left(G_{z}^{\epsilon}\left(\xi_{2}\right)-G_{z}^{\epsilon}\left(\xi_{1}\right)\right)\left(\xi_{2}-\xi_{1}\right)>(\alpha+\epsilon)\left(\xi_{2}-\xi_{1}\right)^{2}$ and (2.15) holds, we see that if $z_{-}$and $y_{-}$ are in $E_{-}$and $z_{+} \in E_{+}$, then

$$
\left(D_{1} h\left(z_{-}, z_{+}\right)-D_{1} h\left(y_{-}, z_{+}\right)\right)\left(z_{-}-y_{-}\right) \leq m_{1}\left\|z_{-}-y_{-}\right\|^{2},
$$

where $m_{1}=1-\frac{\alpha}{j_{1}-1}<0$.

(iii) Similarly, using the fact that $\left(G_{z}^{\epsilon}\left(\xi_{2}\right)-G_{z}^{\epsilon}\left(\xi_{1}\right)\right)\left(\xi_{2}-\xi_{1}\right)<(\beta+\epsilon)\left(\xi_{2}-\xi_{1}\right)^{2}$ and (2.17) holds, we see that if $z_{+}$and $y_{+}$are in $E_{+}$and $z_{-} \in E_{-}$, then

$$
\left(D_{2} h\left(z_{-}, z_{+}\right)-D_{2} h\left(z_{-}, y_{+}\right)\right)\left(z_{+}-y_{+}\right) \geq m_{2}\left\|z_{+}-y_{+}\right\|^{2},
$$

where $m_{2}=1-\frac{\beta}{j_{1}+1}>0$.

(iv) If $\theta(v)$ denotes the unique $\left(z_{-}+z_{+}\right)(v) \in E_{-} \oplus E_{+}$which solves (2.11), then $\theta \in$ $C^{1}\left(E_{0}, E\right)$. In fact, if $v, v^{\prime} \in E_{0}$, and $p_{1}=\theta(v), p_{2}=\theta\left(v^{\prime}\right)$, then we have

$$
\begin{aligned}
\left\|p_{1}-p_{2}\right\|_{E} & =\left\|\left(A_{\epsilon}\right)^{-1}\left(P_{-}+P_{+}\right)\left[F_{\epsilon}\left(v+p_{1}\right)-F_{\epsilon}\left(v^{\prime}+p_{2}\right)\right]\right\|_{E} \\
& \leq C\left\|\left(v+p_{1}\right)-\left(v^{\prime}+p_{2}\right)\right\|_{E} \\
& \leq C\left\|\left(v-v^{\prime}\right)-\left(p_{1}-p_{2}\right)\right\|_{E} .
\end{aligned}
$$

Thus we have

$$
\left\|p_{1}-p_{2}\right\|_{E} \leq \frac{C}{1-C}\left\|v-v^{\prime}\right\|_{E}
$$

Thus $\theta$ is continuous. Since $F_{\epsilon} \in C^{1}(E, E), \theta \in C^{1}\left(E_{0}, E\right)$. Since $\operatorname{dim} L_{0}$ is finite and all topologies on $L_{0}$ are equivalent, we have

$$
\left|A_{\epsilon}\right|^{\frac{1}{2}} z_{+}(v) \in C^{1}\left(L_{0}, L\right) .
$$


Let $v \in E_{0}$. If $q \in E_{-} \oplus E_{+}$, then from (2.11) we have

$$
\int_{0}^{2 \pi}\left[A_{\epsilon}(\theta(v)) \cdot q-\left(P_{-}+P_{+}\right) F_{\epsilon}(v+\theta(v)) \cdot q\right] d t=0 .
$$

Since $\int_{0}^{2 \pi} A_{\epsilon} \nu \cdot q=0$, we have

$$
D I(v+\theta(v))(w)=\int_{0}^{2 \pi}\left[A_{\epsilon}(v+\theta(v)) \cdot q-\left(P_{-}+P_{+}\right) F_{\epsilon}(v+\theta(v)) \cdot q\right] d t=0
$$

for all $q \in E_{-} \oplus E_{+}$.

(v) Since the functional $I$ has a continuous Fréchet derivative $D I, \tilde{I}$ has a continuous Fréchet derivative $D \tilde{I}$ with respect to $v$.

(vi) Suppose that there exists $v \in E_{0}$ such that $D \tilde{I}(v)=0$. From $D \tilde{I}(v)(h)=D I(v+\theta(v))(h)$ for all $v, h \in E_{0}, D I(v+\theta(v))(h)=0$ for all $h \in E_{0}$. Since $D I(v+\theta(v))(w)$ for all $w \in E_{-} \oplus E_{+}$, it follows that $D I(v+\theta(v))=0$. Thus $v+\theta(v)$ is a solution of (1.1). Conversely if $u$ is a solution of (1.1) and $v=P_{0} u$, then $D \tilde{I}(v)=0$.

\section{Proof of Theorem 1.1}

Lemma 3.1 Assume that G satisfies the conditions (G1)-(G5). Then - I( $(v)$ is bounded below and satisfies (P.S.) condition.

Proof Let $v \in E_{0}$. By the finite dimensional reduction,

$$
\tilde{I}(v)=\frac{1}{2}\left(A_{\epsilon}(v+\theta(v)), v+\theta(v)\right)-\int_{0}^{2 \pi} G^{\epsilon}(t, v(t)+\theta(v(t))) d t,
$$

where $\theta(v)=\theta_{-}(v)+\theta_{+}(v), v \in E_{0}, \theta_{-}(v) \in E_{-}, \theta_{+}(v) \in E_{+}, G^{\epsilon}(t, v(t)+\theta(v(t)))=G(t, v(t)+$ $\theta(v(t)))+\epsilon(v(t)+\theta(v(t)))^{2}$. Let $w=v+\theta_{-}(v)$. Then we have

$$
\begin{aligned}
\tilde{I}(v)= & \frac{1}{2}\left(A_{\epsilon}(w), w\right)-\int_{0}^{2 \pi} G^{\epsilon}(t, w(t)) d t \\
& +\left[\frac{1}{2}\left(\left(A_{\epsilon}(\theta(v)), \theta(v)\right)-\left(A_{\epsilon}(w), w\right)\right)-\int_{0}^{2 \pi}\left(G^{\epsilon}(t, \theta(v(t)))-G^{\epsilon}(t, w(t))\right) d t\right] .
\end{aligned}
$$

Moreover, we have

$$
\begin{aligned}
& \frac{1}{2}\left(\left(A_{\epsilon}(\theta(v)), \theta(v)\right)-\left(A_{\epsilon}(w), w\right)\right)-\int_{0}^{2 \pi}\left(G^{\epsilon}(t, \theta(v(t)))-G^{\epsilon}(t, w(t))\right) d t \\
& =-\int_{0}^{2 \pi}\left(G_{z}^{\epsilon}\left(t, s \theta_{+}(v(t))-w(t)\right), \theta_{+}(v(t))\right) d s+\frac{1}{2}\left(A_{\epsilon}(\theta(v)), \theta_{+}(v)\right) \\
& \quad=\int_{0}^{2 \pi} \int_{0}^{2 \pi}\left(d_{z}^{2} G^{\epsilon}\left(t, s \theta_{+}(v(t))+w(t)\right) \theta_{+}(v(t)), \theta_{+}(v(t))\right) s d s d t \\
& \quad-\frac{1}{2}\left(A_{\epsilon}\left(\theta_{+}(v)\right), \theta_{+}(v)\right) \\
& \leq 0
\end{aligned}
$$


By (G4), we have chosen a number $\gamma$ such that $j_{1}<\gamma<d_{z}^{2} G(t, \infty)<\beta$. Thus we have

$$
\begin{aligned}
\tilde{I}(v) & \leq \frac{1}{2}\left(A_{\epsilon}(w), w\right)-\int_{0}^{2 \pi} G^{\epsilon}(t, w(t)) d t \\
& \leq \frac{1}{2}\left(j_{1}-\gamma\right)\|w\|_{L^{2}}^{2}+C \rightarrow-\infty \quad \text { as }\|v\|_{E} \rightarrow \infty
\end{aligned}
$$

Thus $-\tilde{I}(v)$ is bounded from below and satisfies (P.S.) condition.

Lemma 3.2 Assume that $G$ satisfies conditions (G1)-(G5). Then $v=0$ is a strict local point of minimum of $\tilde{I}(v)$ with $\tilde{I}(0)=0$.

Proof

$$
\begin{aligned}
\tilde{I}(v) & =I(v+\theta(v)) \\
& =\frac{1}{2}\left(A_{\epsilon}(v+\theta(v)), v+\theta(v)\right)-\int_{0}^{2 \pi} G^{\epsilon}(t, v(t)+\theta(v(t))) d t \\
& =\frac{1}{2}\left(A_{\epsilon}(v), v\right)+C,
\end{aligned}
$$

where

$$
\begin{aligned}
C= & \frac{1}{2}\left(A_{\epsilon}(\theta(v)), \theta(v)\right)-\int_{0}^{2 \pi} G^{\epsilon}(t, \theta(v(t))) d t \\
& -\int_{0}^{2 \pi}\left[G^{\epsilon}(t, v(t)+\theta(v(t)))-G^{\epsilon}(t, \theta(v(t)))\right] d t \\
= & \tilde{I}(0)-\int_{0}^{2 \pi}\left[G^{\epsilon}(t, v(t)+\theta(v(t)))-G^{\epsilon}(t, \theta(v(t)))\right] d t, \\
\lim _{|v| \rightarrow 0} \tilde{I}(v)-\tilde{I}(0) & =\frac{1}{2}\left(A_{\epsilon}(v), v\right)-\lim _{|v| \rightarrow 0} \int_{0}^{2 \pi}\left[G^{\epsilon}(t, v(t)+\theta(v(t)))-G^{\epsilon}(t, \theta(v(t)))\right] d t \\
& =\frac{1}{2}\left(A_{\epsilon}(v), v\right)-\lim _{|v| \rightarrow 0} \int_{0}^{2 \pi} G_{z}^{\epsilon}(t, s v(t)+\theta(v(t))) v(t) d t .
\end{aligned}
$$

Thus we have

$$
\lim _{|v| \rightarrow 0} \tilde{I}(v)-\tilde{I}(0)=\frac{1}{2}\left(j_{1}-d_{z}^{2} G(t, 0)\right)\|v\|_{L^{2}}>0 .
$$

Thus $v=0$ is a strict local point of minimum of $\tilde{I}(v)$. Since $\theta(0)=0, \tilde{I}(0)=0$.

Proof of Theorem 1.1 By Lemma 2.1(v), $\tilde{I}(v)$ is continuous and Fréchet differentiable in $E_{0}$. By Lemma 3.1, $\tilde{I}(v)$ is bounded above, satisfies the (P.S.) condition and $\tilde{I}(v) \rightarrow-\infty$ as $\|v\|_{E} \rightarrow \infty$. By Lemma 3.2, $v=0$ is a strict local point of minimum of $\tilde{I}(v)$ with a critical value $\tilde{I}(0)=0$. We note that $\max _{v \in E_{0}} \tilde{I}(v)>0$ is another critical value of $\tilde{I}$. By the shape of the graph of the functional $\tilde{I}$ on the one-dimensional subspace $E_{0}$, there exists the third critical point of $\tilde{I}(v)$. Thus (1.1) has at least three solutions, one of which is a trivial solution $u=v+\theta(v)=0+0=0$. 
Competing interests

The authors did not provide this information.

\section{Authors' contributions}

The authors did not provide this information.

\section{Author details}

${ }^{1}$ Department of Mathematics, Kunsan National University, Kunsan, 573-701, Korea. ${ }^{2}$ Department of Mathematics

Education, Inha University, Incheon, 402-751, Korea.

\section{Acknowledgements}

The authors appreciate very much the referees for their kind corrections. This work (Tacksun Jung) was supported by Basic Science Research Program through the National Research Foundation of Korea (NRF) funded by the Ministry of Education, Science and Technology (KRF-2011-0026920).

Received: 20 August 2012 Accepted: 9 February 2013 Published: 5 March 2013

\section{References}

1. Chang, KC: Infinite Dimensional Morse Theory and Multiple Solution Problems. Birkhäuser, Boston (1993)

2. Jung, T, Choi, QH: On the number of the periodic solutions of the nonlinear Hamiltonian system. Nonlinear Anal. TMA 71(12), e1100-e1108 (2009)

3. Jung, T, Choi, QH: Periodic solutions for the nonlinear Hamiltonian systems. Korean J. Math. 17(3), 331-340 (2009)

4. Rabinowitz, PH: Minimax Methods in Critical Point Theory with Applications to Differential Equations. CBMS Regional Conf. Ser. Math., vol. 65. Am. Math. Soc., Providence (1986)

doi:10.1186/1029-242X-2013-91

Cite this article as: Jung and Choi: At least three solutions for the Hamiltonian system and reduction method. Journal of Inequalities and Applications 2013 2013:91.

\section{Submit your manuscript to a SpringerOpen ${ }^{\circ}$ journal and benefit from:}

- Convenient online submission

- Rigorous peer review

- Immediate publication on acceptance

- Open access: articles freely available online

- High visibility within the field

- Retaining the copyright to your article 